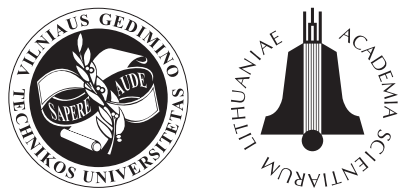

TRANSPORT

2010

25(4): 433-441

\title{
ESTIMATION OF THE LOGIT MODEL FOR THE ONLINE CONTRAFLOW PROBLEM
}

\author{
Habibollah Nassiri ${ }^{1}$, Ali Edrissi ${ }^{2}$, Hamed Alibabai ${ }^{3}$ \\ 1, 2 Dept of Civil Engineering, Sharif University of Technology, Azadi Ave, 11155-9313 Tehran, Iran \\ ${ }^{3}$ Transportation Centre, Northwestern University, 600 Foster Street, Evanston, IL 60201, USA

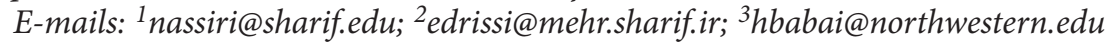

Received 5 February 2010; accepted 15 November 2010

\begin{abstract}
Contraflow or lane reversal is an efficient way for increasing the outbound capacity of a network by reversing the direction of in-bound roads during evacuations. Hence, it can be considered as a potential remedy for solving congestion problems during evacuation in the context of homeland security, natural disasters and urban evacuations, especially in response to an expected disaster. Most of the contraflow studies are performed offline, thus strategies are generated beforehand for future implementation. Online contraflow models, however, would be often computationally demanding and time-consuming. This study contributes to the state of the art of contraflow modelling in two regards. First, it focuses on the calibration of a Logit choice model which predicts the online contraflow directions of strategic lanes based on the set of directions obtained from offline scenarios. This is the first effort to adjust offline results to be applied for an online case. The second contribution of this paper is the generation of calibration data set from a novel approach through simulation. The calibrated Logit model is then tested for the network of the City of Fort Worth, Texas. The results show a high performance of this approach to generating beneficial strategies, including an increase in up to $16 \%$ in throughput compared to no contraflow case.
\end{abstract}

Keywords: contraflow, evacuation, Logit model, online application, throughput, bi-level programming.

\section{Introduction}

Natural and man-made disasters have been a major concern to humanity and a threat to lives and property. Although little can be done to prevent disasters, there are actions that can reduce their impacts. Transportation and logistic systems are two of the most critical infrastructures during disasters. Managing traffic under such difficult and uncertain circumstances is a complicated and challenging task and requires special actions such as reversing lanes, reassigning transit routes and regularly updating.

Among disaster response actions, evacuation has received meticulous attention in literature. As one of the cornerstones in the response phase of emergency management, evacuation refers to the movement of populations from a dangerous place to a safe refuge. The effective control of traffic resulting from evacuation has emerged as one of the critical research issues in the transportation community, mainly due to complexities such as unexpectedly high demand surge and restricted network capacity.

There have been various studies incorporating different approaches for modelling the evacuation problem.
The idea of finding paths minimizing travel distance and travel time or maximizing the expected number of evacuees within a given time has been considered in many studies (Dunn and Newton 1992; Miller-Hooks and Patterson 2004; Miller-Hooks and Sorrel 2008). Some studies have incorporated the concept of lane-based routing to reduce intersection delays (Cova and Johnson 2003). This is accomplished by temporarily transforming intersections into uninterrupted flow facilities through proper turning restrictions. Nuclear power plant evacuation is another area of concern which has appeared in some studies (Campos et al. 1999). The idea of the stochasticity of demand and supply has been brought into the study of evacuation (Smith 1991; Talebi and Smith 1985) in which evacuation plans are built using a statedependent queuing model. On the demand control side of the evacuation problem, the idea of simultaneous and staged evacuation was proposed by some researches (Chen and Zhan 2008). There are some studies presenting approaches to model demand and supply for shortnotice evacuations. In these cases, persons may need to be evacuated directly from their current location. For some disasters, the spatial extent of the evacuated area may change over time (Noh et al. 2009). In another study, 
the evacuation scheduling problem was formulated as a mathematical programming model to minimize the total travel time by controlling demand (Chiu 2004). The idea to concurrently optimize destination/route choice and zone scheduling with demand mobilization duration has also been tested through a mixed-integer linear programming model (Tuydes 2005). Recently, dynamic traffic assignment models have been included in evacuation studies to capture a dynamic equilibrium seeking the behaviour of drivers. As an example, Sbayti and Mahmassani (2006) propose a bi-level program which uses dynamic traffic assignment at a lower level and minimizes clearance time at a higher level. In another study, a simulation-optimization framework is proposed to find the system optimal solution for the problem of emergency evacuation planning (Afshar and Haghani 2008). The model simultaneously solves the choice of destination (shelter), evacuation route and departure time for all evacuees from the given origins (endangered zones). In this respect, a heuristic algorithm is proposed to find the assignment of system-optimal dynamic traffic during an iterative procedure. In another effort, a strategic dynamic traffic assignment model of hurricane evacuation was developed (Brown et al. 2009). In addition, to minimize the run time of the model and to maximize the capabilities of policy analysis, this model can be applied to compare the relative system and evacuation corridor performance of alternative policy scenarios.

Contraflow is a useful tool for facilitating the evacuation process and managing traffic. Usually, the term 'contraflow' is used to refer to the reversal of lanes which are normally only configured for travelling in one direction. The issues of assessing the alternative entrance and termination designs of contraflow segments in evaluating the hurricane evacuation plan for cities has been studied by some researchers (Wolshon 2001, 2002; Lim and Wolshon 2005). The critical design of contraflow entry points was performed using simulation software DYNASMART-P to test alternative plans for evacuating downtown Minneapolis (Kwon and Pitt 2005). Link-coupling techniques for contraflow design matches network segments that can exchange capacity in case of reversing (Tuydes 2005). When using this technique, lane-based capacity reversibility and total-or-no-capacity reversibility replace the continuous variable commonly seen in the evacuation problem (Tuydes 2005). Moreover, to cope with high computational cost associated with analytical Dynamic Traffic Assignment (DTA) formulations, a heuristic algorithm using both simulation-assignment and Tabu Search methods have been proposed for potential application in real-life large-scale evacuation networks (Tuydes and Ziliaskopoulos 2006). The dynamic capacity reallocation problem using simulation software package DYNASMART can generate a time-dependent contraflow control policy to be deployed at target links during a major evacuation (Sbayti 2008).

Due to the difficulty level of the contraflow problem and intrinsic uncertainty inherent in disasters, it is impractical to determine optimal online contraflow strategies for a large network in a short period of disaster.
Therefore, there is a critical need for finding the optimal direction in an online scenario based on the findings from offline scenarios. This leads the researchers to seek for semi-optimal lane reversal strategies providing a balance between accuracy and computational time. Literature shows a few studies on online traffic management regarding disaster situations. In this respect, some studies focus on an adaptive control framework for real-time evacuation traffic management using DTA approach (Liu et al. 2007) while others compare different types of routing including: static routing that dispatches vehicles to different routes only at their departures and dynamic routing where vehicles can be assigned to a new route based on the prevailing network conditions (Yuan et al. 2006). Also, based on reference (Chiu 2004), real-time traffic management models are able to influence system performance towards an optimum level. These models focus on the route choice decisions made by evacuees and a real-time pre-trip route guidance scheme. To sum up, although many studies have mentioned the online contraflow problem as an important issue, none of those have addressed it thoroughly.

In this paper, the Logit choice model predicts the contraflow direction of strategic lanes in the online scenarios based on their direction in the offline scenarios which are the cases of the complete disruption of a single link in the network while online scenarios are the cases of the disruption of two or more links of the network. It should be noted that the links may not be completely disrupted in the online scenarios. The offline cases are countable (i.e. the number of the offline cases are equal to the number of the links in the network), so the contraflow problem can be solved for all of the links. On the other hand, the online scenarios are not countable and finding a solution for all of them is not practical. In this paper, the assumption that a contraflow solution to the online scenario can be obtained from the contraflow solution to its constituent offline scenarios is tested, i.e. the direction of the strategic lane in the online scenario is taken from its optimal direction in one of the constituent offline scenarios based on a choice model. The paper will evaluate the validity of this assumption through testing the results of the choice model. A novel approach has been exploited to obtain the calibration data set from simulation explained throughout the paper.

The rest of this paper is organized as follows: the next section explains the modelling framework in detail; section 3 considers the calibration problem and data collection methodology done through a novel approach from simulation. The case study is introduced in section 4 followed by numerical results in section 5 . Section 6 summarizes the results and concludes the paper.

\section{Modelling Framework}

Contraflow strategies are required for calibrating the Logit choice model and are obtained through an assignment-based bi-level program which uses DYNASMART-P software as its assignment tool. DYNASMART-P (Mahmassani 2000; Mahmassani et al. 2005) 
is dynamic traffic assignment software based on an mesoscopic simulation approach modelling individual vehicle movements in the network and updating path flows in the equilibration process. The calibration data set is created with running contraflow problems for different link disruption scenarios and the calibrated Logit model along with the Monte Carlo simulation approach predicts efficient contraflow strategies for strategic lanes in online scenarios based on their direction in constituent offline scenarios. The details of the prediction process are clarified in the section called the adjustment of the offline results.

\subsection{Contraflow Problem}

For the purpose of the study, contraflow is modelled through the bi-level programming approach. Bi-level programming is a hierarchical mathematical problem where the set of all variables is partitioned between vectors $x$ and $y$. Given $y$, vector $x$ is to be chosen as the optimal solution $x=x(y)$ of an optimization problem parameterized in $y$ :

$$
X(y) \in \varphi(Y):=\underset{x}{\operatorname{Arg} \min }\{f(x, y): g(x, y) \leq 0\} .
$$

This problem is called the lower level problem or the follower's problem. Solution $x(y)$ is called the rational reaction of the follower on leader's choice $y$. Knowing this reaction the bi-level problem can be read as follows:

$$
\underset{y}{\operatorname{Min}}\{F(x, y): G(y) \leq 0, x \in \varphi(y)\},
$$

which is the master problem of the bi-level problem.

\subsection{Master Problem}

The master (upper level) problem determines the optimal reversing of the strategic lanes of the network links in order to maximize throughput defined as the number of the evacuated vehicles within evacuation time $T$, which is an input of the algorithm. Those not able to exit the network within $T$ are considered as victims. The mathematical formulation of the master problem is given in Eq. (3):

$$
\begin{aligned}
& \operatorname{Max}(T P) \\
& \text { subjected to: } \\
& \begin{array}{l}
T P=\sum_{p} \lambda_{p}^{T}, \\
\lambda_{p}^{T}=f\left(\vartheta, h_{p}^{t} \quad \forall p, t\right),
\end{array}
\end{aligned}
$$

where: TP is the throughput of the network within the period of time $T ; \lambda_{p}^{T}$ is accumulative completed trips on route $p$ within the period of time $T$ (which is an output of the traffic assignment problem); $h_{p}^{t}$ is a departed flow from route $p$ at time $t ; \vartheta$ is network configuration that includes lane directions.

The solution to optimal contraflow design for maximum throughput is obtained using the Genetic Algorithm. Based on the performance of two sets of lane configurations (i.e. two contraflow strategies), the new sets of contraflow strategies are generated until a stop criterion is satisfied. The stop criterion is satisfied if in 10 consecutive generations a better solution (i.e. higher throughput) is not achieved or a maximum of 150 iterations is reached. A strategic lane is defined as a lane having an alterable direction. Each strategic lane has two possible states, i.e. directions. Thus, each strategic lane can be considered a gene and the length of one chromosome is equal to the number of strategic lanes. Therefore, when there are $M$ strategic lanes, each solution is a chromosome of length $M$ (i.e. includes $M$ genes). The following parameters were used for the Genetic Algorithm:

- crossover type $=$ single breakpoint;

- crossover probability $=0.4$;

- mutation types = bit inversion;

- mutation probabilities $=0.02$;

- population $=16$.

Considering each of the iterations of the Genetic Algorithm, a set of new feasible lane configuration (new chromosome) is generated. For more discussion on the Genetic Algorithm one can refer to (Davis 1991).

\subsection{Lower Level Problem}

As seen in Eq. (3), a set of variables $\lambda_{p}^{T}$ are the results of a function of path flows and network configuration. This function is the so called dynamic traffic assignment problem. A simulation-based dynamic traffic assignment is an alternative approach replacing an analytical dynamic traffic assignment. The main advantage of using simulation is capturing the complex traffic phenomena such as FIFO condition and queue spillback in congested intersections. With the later approach, the complexity of traffic is captured by a traffic simulation model and then an optimization algorithm renews route choice variables. DYNASMART-P (Mahmassani 2000; Mahmassani et al. 2005) as our assignment software has been exploited in this study to solve the lower level problem. DYNASMART-P is able to solve user equilibrium and system optimum assignment for multi user classes. In this work, the System Optimum (SO) dynamic traffic assignment feature of DYNASMART-P has been exploited to preclude the occurrence of the well known braes paradox. Also, in the presence of emerging informing technologies, using system optimal conditions are quite reasonable. The assignment method consists of the following steps:

1. Finding an initial solution $\left(h_{p}^{t} \forall p, t\right)$.

2. Loading these flows with a traffic simulator.

3. Computing marginal travel times $\left(T_{p}^{t} \forall p, t\right)$.

4. Finding a new solution point $\left(h_{p}^{t} \forall p, t\right)$.

5. Checking the stopping criterion. If the criterion is satisfied - stop; otherwise go to step 2. The stopping criterion is selected as the number of OD pairs with a travel time gap of more than 0.5 seconds which is less than 10 .

The bi-level approach is applied in the following way. First, DYNASMART-P is run with the initial population of lane configurations. Throughputs are computed and plugged into the master problem. New lane configuration in the master problem is created and plugged again as an input to DYNASMART-P. This procedure is repeated until convergence. 


\subsection{Adjustment of the Offline Results}

As mentioned previously, the focus of this study is on the prediction of online scenario contraflow configurations based on a set of offline scenario contraflow configurations. What is referred to as the offline scenario is a case of the total disruption (100\% capacity reductions) of the single links of the network. Therefore, the total number of the offline scenarios is equal to the number of the links in the network. On the other hand, the online scenario is a scenario where more than one link has been disrupted at the same time. Also, it is possible that each of these links has been disrupted in different degrees of severity (different levels of Capacity Reduction $(C R))$. As the number of multiple breakdowns in the network is unlimited because of different combinations in the links and levels of capacity reduction, finding the optimal solution is impossible in all these cases.

This study overcomes this failure by predicting online contraflow directions from offline contraflow directions. Each online scenario consists of some offline scenarios and the Logit choice model determines from which of these offline scenarios the direction of each strategic lane should be taken. The selection is based on the attributes of the disrupted links in the online scenario justifying the selection procedure. This assumption is tested through analyzing the result of the choice model.

As an example, assume that there is a mixed disruption scenario in which two arbitrary links $A$ and $B$ have been broken down at $C R_{A}$ and $C R_{B}$ levels of capacity reduction respectively. As mentioned before, the contraflow direction of each strategic lane in the mixed disruption scenario (disruption of both links $A$ and $B$ ) is the same as its direction in one of the scenarios of the disruption of $A$ or $B$. This choice is made using the Logit choice model calculating the probabilities of choosing the lane direction from lane directions in one of the two offline scenarios. The utility function of selecting from scenarios $A$ or $B$ is defined as follows:

$$
U_{X}=a \times\left(C R_{X}\right)+b \times\left(I M_{X}\right) ; X=A \text { or } B .
$$

Taking into consideration the problem of choice making, the choice set is:

- obtaining the direction of the strategic lane from its direction in the scenario of the first lane $(A)$ breakdown;

- obtaining the direction of the strategic lane from its direction in the scenario of the second lane (B) breakdown.

The parameters of the model are assumed to be generic as $A$ and $B$ are not actual links but labels. Also, as the choice model is a physical model rather than the behavioural one, the choices are independent. In the utility function, variable $C R_{X}$ is the percentage of the capacity reduction of link $X$ with respect to the normal state of no disruption and $M_{X}$ is 'Birnbaum probabilistic importance measure of link $X$ ' (a scale of 1 to 6 for $I M_{x}$ has been used) calculated as follows:

$$
I M_{X}=\frac{\partial T P(p)}{\partial P_{X}}=T P\left(1_{X}, p\right)-T P\left(0_{X}, p\right) .
$$

Let $T P(p)$ denote system throughput as a function of link reliability vector $p=\left\{p_{1}, p_{2}, p_{3}, \ldots, p_{n}\right\}$ in which $\mathrm{n}$ is the number of the links in the network. Conditional system throughput given that specific component $X$ fails or not and can be written as $\operatorname{TP}\left(0_{X}, p\right)$ or $T P\left(1_{X}, p\right)$ respectively. The difference between these two cases is referred to as 'Birnbaum probabilistic importance measure' that implies the probabilistic contribution of improving component reliability to that of system throughput as discussed in Birnbaum (1968).

The proof is very simple. According to the theorem of total probability, system throughput $T P(p)$ can be written as a linear function in terms of $P_{X}$ and the probability of the disruption of link $X$ is:

$$
T P(p)=p_{X} \cdot T P\left(1_{X}, p\right)+\left(1-p_{X}\right) \cdot T P\left(0_{X}, p\right) .
$$

Eq. (5) is obtained by differentiating both sides of Eq. (6) with respect to $p_{X}$.

In order to calculate $I M_{X}$, throughput should be calculated from two different scenarios, with and without link $X . I M_{X}$ is then calculated from Eq. (5) and scaled into the range of 1 to 6 .

As a limit case example, let's assume that the first link (link $A$ ) is disrupted in $100 \%$ and has a degree of importance 6 (maximum degree of importance), whereas the second link (link B) is only slightly disrupted and has a degree of importance 1 . In this case, most probably, the contraflow directions of strategic lanes are taken from that of the scenario of the single disruption of the first link.

For each strategic lane, direction is selected through the Monte Carlo simulation approach. Using the Logit model, the first probability of choosing lane direction from scenario $A$ or $B$ is calculated based on Eq. (7):

$$
P_{A}=\frac{e^{U_{A}}}{e^{U_{A}}+e^{U_{B}}} ; \quad P_{B}=1-P_{A} .
$$

Afterwards, random number $(\mathrm{U})$ is generated from a linear congruent generator. If $0<U<P_{A}$, lane direction is taken from the scenario of disruption $A$. Otherwise, the direction is taken from the scenario of disruption $B$. The same procedure is repeated for all strategic lanes.

The extendibility of the results of two disruption cases to more than two disruption cases is straightforward as a result of independence from irrelevant alternatives (IIA) properties of Logit models. Also, other traffic or geometric design variables may be used in the utility function.

\section{Calibration}

The calibration of the Logit choice model is done based on the data set obtained through a novel approach from simulation. A sample disaggregate dataset generated through simulation is shown in Table 1 where each record is related to one scenario. To obtain each record, three contraflow problems, including a scenario of disrupting only link $A$, a scenario of disrupting only link $B$ and a mixed disruption scenario need to be solved. As 
shown in Table 1, optimal lane configuration for each of these scenarios is reported in columns 4,7 and 8 respectively. Each of these columns is a chromosome of length 4 as there are four strategic lanes in this imaginary network. The other columns show the attributes of the contraflow scenario. A dataset of Table 1 is converted into the format of Table 2 . This conversion simply assigns a record number to each strategic lane of each scenario in Table 1. In this table, each record is related to a strategic lane of each of the scenarios. Therefore, the number of records in Table 2 would be four times of the number of records in Table 1. However, it could be different because during conversion from Table 1 to Table 2, the following steps are applied:

1. if strategic lane direction in the mixed scenario does not agree with any of single breakdown scenarios, neglect this record;

2. if lane direction in both of single breakdown scenarios agrees with lane direction in the mixed scenario, add two records - one for each of the single disruption cases chosen;

3. if single breakdown solutions are not the same, one record is added to the converted table.

Thus, Table 2 shows that the first five columns are the same as Table 1. Columns 4, 7 and 8 indicate the direction of the strategic lane in three scenarios. In this table, $y_{A}=1$ means the direction of the strategic lane in the mixed scenario and is chosen from its direction in scenario $A$ and $y_{B}=1$ means the direction chosen from scenario $B$.

Table 2 shows that $C R_{A}, I M_{A}, C R_{B}$, and $I M_{B}$ are taken from record 1 of Table 1 as these lanes are taken from scenario 1 . The first record is related to the first gene of the chromosome of scenario 1. Lane direction in the mixed scenario is selected from direction in scenario $B$.
The next two records are related to the second gene of the chromosome of scenario 1. Because both individual solutions agree with the mixed solution, two records have been added to Table 2 . In one of these records, $y_{A}$ is a set equal to 1 , whereas in the other, $y_{B}$ is a set equal to 1 . The fourth record is related to the third gene of the chromosome of scenario 1 . In this record, lane direction in the mixed scenario is selected from direction in scenario $B$. The fourth gene of the chromosome of scenario 1 is neglected as a mixed solution does not agree with any of the individual solutions.

By performing this conversion, a disaggregate dataset is obtained and used as input to the maximum likelihood estimator.

\section{Case Study}

The street network of the City of Fort Worth, Texas is selected for this study. The City is the fifth-largest city in Texas and the 18th largest city in the United States covering nearly 300 square miles. Fort Worth has a population of approximately 653320. Fig. 1 shows the Fort Worth network, including the node numbers and zone boundaries of this network. The network consists of 180 traffic nodes, 445 links and 13 zones. Traffic demand is generated from each of the zones and vehicles leave their origin node heading toward the safe spot located in zone 2 that is at the ultimate bottom zone of the network and consists of single node 117. Demand distribution from zone 1 through zone 13 to zone 2 are 10659, 795, 2054, 1865, $3055,3220,1223,2201,2952,1964,2025,1902$ and 3891 respectively. These vehicles depart uniformly throughout the first 5 minutes of the simulation. This study examines 403 strategic lanes located on 227 links and coloured red and blue in Fig. 1. The clearance time of the strategic lanes is considered to be zero, i.e. the network is assumed

Table 1. A sample database before conversion

\begin{tabular}{|c|c|c|c|c|c|c|c|c|c|c|c|c|c|c|c|c|}
\hline \multirow{2}{*}{$\begin{array}{c}\begin{array}{c}\text { Record } \\
\text { number }\end{array} \\
1 \\
\end{array}$} & \multirow{2}{*}{$\begin{array}{c}C R_{A} \\
70 \\
\end{array}$} & \multirow{2}{*}{$\frac{I M_{A}}{4}$} & \multicolumn{4}{|c|}{$\begin{array}{l}\text { Contraflow solution } \\
\text { for } A\end{array}$} & \multirow{2}{*}{$\frac{C R_{B}}{30}$} & \multirow{2}{*}{$\frac{I M_{B}}{4}$} & \multicolumn{4}{|c|}{$\begin{array}{c}\text { Contraflow solution } \\
\text { for } B\end{array}$} & \multicolumn{4}{|c|}{$\begin{array}{c}\text { Mixed contra flow } \\
\text { solution }\end{array}$} \\
\hline & & & 0 & 0 & 1 & 1 & & & 1 & 0 & 0 & 1 & 1 & 0 & 0 & 0 \\
\hline 2 & 40 & 3 & 0 & 1 & 1 & 0 & 40 & 3 & 0 & 0 & 1 & 1 & 1 & 1 & 0 & 0 \\
\hline 3 & 60 & 5 & 1 & 0 & 0 & 1 & 40 & 4 & 1 & 0 & 1 & 1 & 1 & 0 & 0 & 0 \\
\hline 4 & 70 & 1 & 0 & 1 & 0 & 0 & 100 & 4 & 1 & 0 & 0 & 1 & 1 & 1 & 1 & 0 \\
\hline 5 & 10 & 2 & 0 & 1 & 1 & 1 & 50 & 2 & 1 & 1 & 0 & 0 & 1 & 0 & 0 & 1 \\
\hline
\end{tabular}

Table 2. A sample database after conversion (the calibration database)

\begin{tabular}{ccccccccccc}
\hline $\begin{array}{c}\text { Record } \\
\text { number }\end{array}$ & $C R_{A}$ & $I M_{A}$ & $C R_{B}$ & $I M_{B}$ & $A$ code & $B$ code & $\begin{array}{c}\text { Mixed } \\
\text { code }\end{array}$ & $y_{A}$ & $y_{B}$ \\
\hline 1 & 70 & 4 & 30 & 4 & 0 & 1 & 1 & 0 & 1 \\
\hline 2 & 70 & 4 & 30 & 4 & 0 & 0 & 0 & 1 & 0 \\
\hline 3 & 70 & 4 & 30 & 4 & 0 & 0 & 0 & 0 & 1 \\
\hline 4 & 70 & 4 & 30 & 4 & 1 & 0 & 0 & 0 & 1 \\
\hline 18 & 10 & 2 & 50 & 2 & 0 & 1 & 1 & 0 & 0 \\
\hline 19 & 10 & 2 & 50 & 2 & 1 & 0 & 0 & 1 \\
\hline 20 & 10 & 2 & 50 & 2 & 1 & 0 & 1 & 0 \\
\hline
\end{tabular}


to be empty at the start of evacuation performed in a short notice choosing evacuation time $(T)$ to be half an hour which considered short in literature on evacuation (Chiu 2004). The results of contraflows are only used for calibrating the Logit choice model.

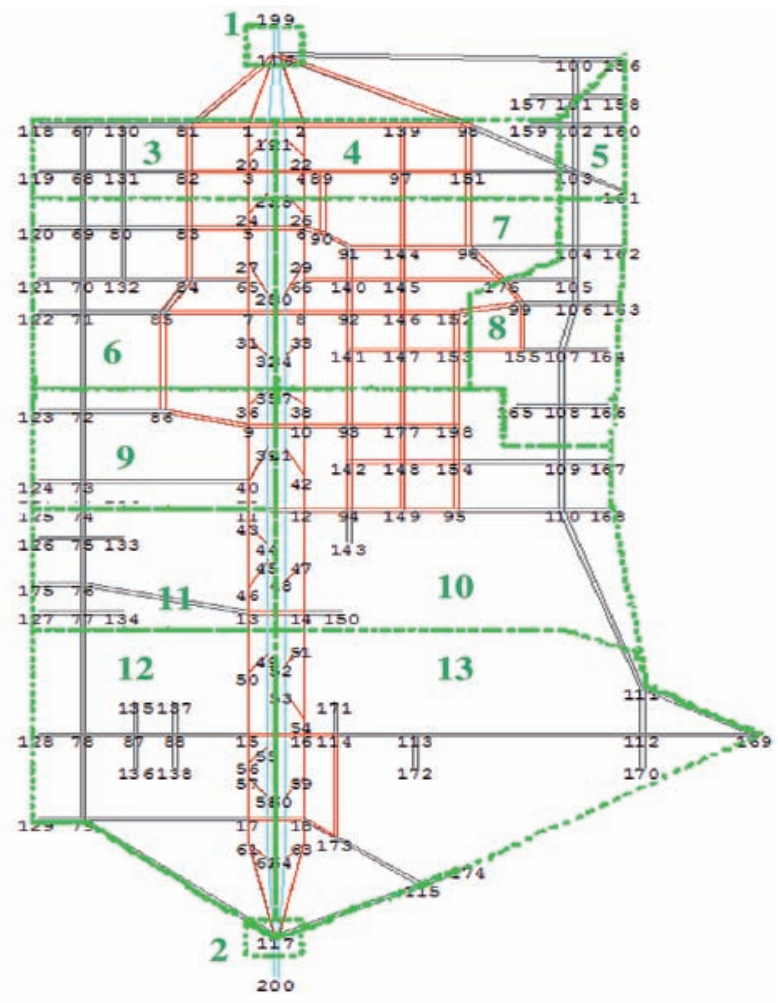

Fig. 1. Fort Worth network (strategic lanes are coloured red and blue) and zone boundaries (coloured green)
In order to calibrate the Logit model, 20 random disruption scenarios were generated in each of which two links were broken down. The disrupted links are selected from 100 most congested links of the network. The details of these scenarios are shown in Table 3. In the starting solution of the contraflow problem, all vertical strategic lanes are set downward as the safe spot is located at the bottom of the network. For each scenario, three contraflow problems need to be solved in order to generate the dataset for the Logit model. After calibration, the model is tested for a different set of 20 scenarios the results of which are reported in the following section.

\section{Numerical Results}

In this section, the results of contraflow for the first scenario introduced in Table 3 are reported. Then, the Logit model and calibration results are presented following the results of testing the built model.

\subsection{Contraflow of Scenario 1}

In scenario 1, link 83-82 and link 70-71 have been disrupted each with $34 \%$ reduction in capacity. These links have a measure of importance making 1 and 1.1 respectively. The contraflow problem stopped following 89 iterations. Fig. 2 shows the trend of changes in throughput considering different iterations. The reason for ascending the trend in throughput is that the current solution in the Genetic Algorithm iterations is always changed to a better solution or is kept unchanged. The optimal throughput after 89 iterations is 13286 vehicles in 30 minutes (evacuation time).

Table 3. Calibration scenarios

\begin{tabular}{|c|c|c|c|c|c|c|}
\hline $\begin{array}{l}\text { Scenario } \\
\text { No. }\end{array}$ & $\begin{array}{c}\text { Disrupted link }(A) \\
\text { (starting node-ending node) }\end{array}$ & $C R_{A}$ & $I M_{A}$ & $\begin{array}{c}\text { Disrupted link }(B) \\
\text { (starting node-ending node) }\end{array}$ & $C R_{B}$ & $I M_{B}$ \\
\hline 1 & $83-82$ & 34 & 1 & $70-71$ & 34 & 1.1 \\
\hline 2 & $82-131$ & 40 & 2.1 & $70-121$ & 30 & 1.9 \\
\hline 3 & $79-78$ & 15 & 3.5 & $86-72$ & 11 & 2.6 \\
\hline 4 & $107-155$ & 23 & 1.5 & $128-78$ & 10 & 1 \\
\hline 5 & $61-62$ & 30 & 2.5 & $74-75$ & 15 & 2 \\
\hline 6 & $76-77$ & 10 & 2 & $68-69$ & 50 & 2.2 \\
\hline 7 & $35-36$ & 67 & 5 & $73-74$ & 40 & 2 \\
\hline 8 & $57-58$ & 45 & 2 & $56-57$ & 30 & 2.7 \\
\hline 9 & $32-35$ & 55 & 4.5 & $39-44$ & 50 & 4.5 \\
\hline 10 & $49-55$ & 65 & 5 & $44-45$ & 70 & 5 \\
\hline 11 & $84-85$ & 20 & 3 & $131-68$ & 23 & 2.3 \\
\hline 12 & $62-117$ & 75 & 6 & $58-62$ & 80 & 5 \\
\hline 13 & $90-6$ & 38 & 3.1 & $99-155$ & 40 & 1 \\
\hline 14 & $55-58$ & 40 & 5 & $78-79$ & 47 & 2 \\
\hline 15 & $116-19$ & 47 & 4 & $23-28$ & 60 & 4 \\
\hline 16 & $77-78$ & 20 & 3.5 & $43-44$ & 25 & 2 \\
\hline 17 & $31-36$ & 30 & 3 & $11-43$ & 20 & 3 \\
\hline 18 & $28-32$ & 50 & 5 & $75-76$ & 60 & 2.5 \\
\hline 19 & $45-49$ & 60 & 5 & 19-23 & 20 & 4 \\
\hline 20 & $25-21$ & 20 & 1 & $165-108$ & 22 & 1 \\
\hline
\end{tabular}




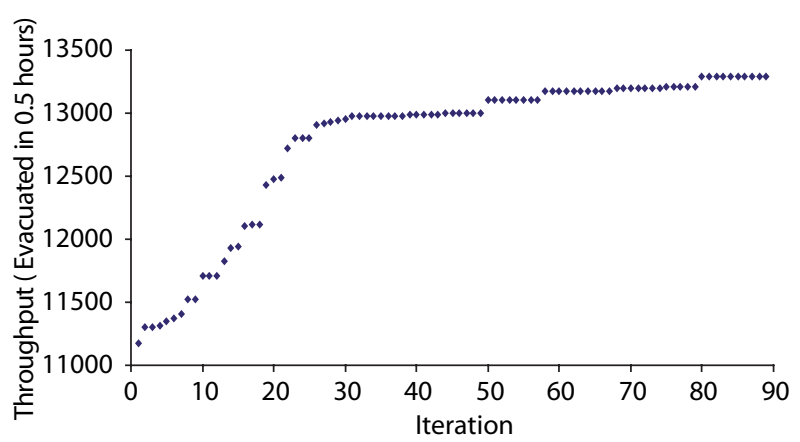

Fig. 2. The trend towards throughput change in the contraflow of scenario 1

\subsection{Calibration of the Model}

As indicated previously, the Logit model was calibrated based on the dataset collected from 20 random scenarios generated from simulation. Accordingly, the number of observation is 8060 (403 strategic lanes $\times 20$ scenarios). $A$ and $b$ respectively as the coefficients of two variables of capacity reduction and the measure of importance are presented in Eq. (4) and estimated to be 0.37 and 0.09 using a maximum likelihood estimation approach of Statistical Package for Social Sciences (SPSS) software. Variables having a positive sign show an increase in the utility function.

Due to the above provided definition, $a$ and $b$ have reasonable signs and are significant at 95\% confidence interval as shown in Table 4. Other statistics are reported in Table 5. The value of $\rho^{2}$ (explanatory power of the model) is reported to be 0.50 which shows improvement regarding the model with respect to having no model. Also, improvements considering the model can be more justified using the chi square test in the following way:

$$
\begin{aligned}
& \chi^{2}=-2(L(0)-L(\beta))=-2(-5375-(-2684))=5381 \\
& \chi_{k=2, p=0.001}^{2}=13.82 ; 5381>13.82 \text { o.k., }
\end{aligned}
$$

which shows improvement in the model with respect to the case of no model with the confidence interval making more than 0.999 . The correlation coefficient between two parameters $(0.33)$ is a significant confidence interval of $95 \%$.

Table 4. The estimated Logit model coefficients

\begin{tabular}{ccc}
\hline Coefficients & Estimated coefficient & Significance \\
\hline $\mathrm{a}$ & 0.37 & 0.04 \\
\hline $\mathrm{b}$ & 0.09 & 0.03 \\
\hline
\end{tabular}

Table 5. Statistics on the calibration Logit model

\begin{tabular}{cc}
\hline Value & Variable \\
\hline 8060 & $N=$ No. of observations \\
\hline-5375.53 & $L(0)$ \\
\hline-2684.74 & $L(\beta)$ \\
\hline-3210.33 & $L(c)$ \\
\hline 0.50 & $\rho^{2}$ \\
\hline 0.16 & $\rho_{c}^{2}$
\end{tabular}

\subsection{Testing the Model}

After calibrating the model based on the random dataset, the calibrated Logit model is evaluated regarding another random dataset from 20 different scenarios also generated from the simulation. For each of these random scenarios, the mixed solution was computed from both the contraflow algorithm and the proposed Logit model. Fig. 3 shows the values of throughput from three different approaches, including optimal, semi optimal (proposed methodology) and no contraflow strategy.

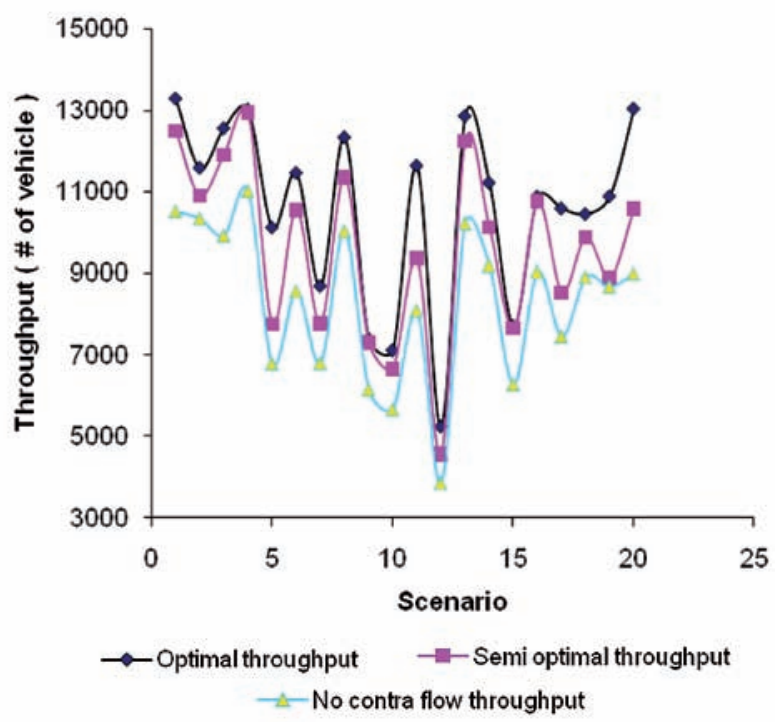

Fig. 3. A comparison of the throughput of three different approaches

As shown in Fig. 3, semi optimal throughputs are close and comparable to optimal throughputs while they are much higher (on average $16 \%$ higher) than no contraflow throughputs which supports adopting the Logit model instead of no contraflow decision during disruptions. Fig. 4 shows optimal throughput and semi optimal throughput plotted against each other. The ideal graph should be a 45 degree sloped line passing through origin, $R$ square of which should be equal to one.

According to Fig. 4, the line has a slope of 0.83 and the interception of 1107 . These numbers are then tested

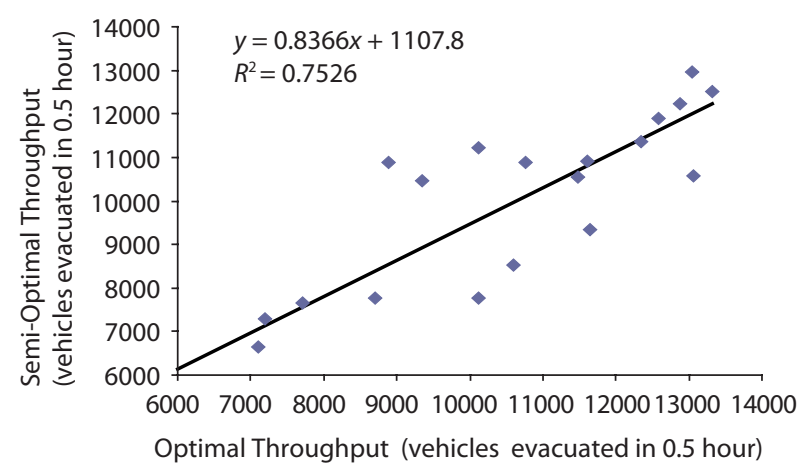

Fig. 4. Optimal throughput versus semi-optimal throughput 
performing t test to ensure they are close enough to one and zero respectively. The results of t test are reported in Table 6 where the hypothesis of $\alpha$ equal to 1 is not rejected at the significance level of 0.16 . In the same way, the hypothesis of $\beta$ equal to 0 is not rejected at the significance level of 0.18 . The value of $R^{2}$ is calculated to be 0.75 which is also in support of the fact that throughput from two approaches lies on a line.

Table 6. A statistical analysis of the throughput regression line

\begin{tabular}{cccccc}
\hline$\alpha$ & $t_{\alpha}$ & $\alpha$ Significance & $\beta$ & $t_{\beta}$ & $\beta$ Significance \\
\hline 0.83 & -1.37 & 0.16 & 1107 & 1.29 & 0.18 \\
\hline
\end{tabular}

Table 7 reports the summary of predicting the direction of the calibrated model on both the calibration dataset and test dataset. For the calibration dataset, the percentage of a correct prediction for lane direction is calculated as $73.5 \%$. The numbers 0 and 1 in this table refer to two different directions of the lane. The table also reports the results of predicting lane direction for the evaluation dataset. In this respect, $58.4 \%$ of lane direction predictions are correct. It is logical that the reported result for the calibration dataset shows a higher percentage of being correct because the model has been calibrated based on this dataset. Also, due to the nature of dynamic traffic assignment, an equilibrium solution is not necessarily unique if existing at all (Szeto and Hong 2006). Hence, the correct percentage of lane direction can be significantly different as it is related to equilibrium flows. This supports findings suggested in Table 7.

Lane directions can be obtained in a couple of seconds using the Logit model compared to the contraflow problem that can take many hours for large networks. This means a significant amount of time saving with a considerable consistency level.

\section{Conclusions}

Contraflow is a widely accepted method for developing efficient evacuation plans when disasters occur. By reversing the direction of lanes in the network, operators facilitate the evacuation process. Disasters are extreme conditions which can affect network capacities by disrupting some of its links and creating the need for op- erators to build efficient plans for evacuating passengers. Building efficient online contraflow plans for evacuating people is of great interest. However, computational requirements for contraflow implementation prohibit traffic management centres from determining optimal contraflow directions online. Also, since there are numerous possible scenarios, planning all them in advance is not practical. Finding semi-optimal contraflow plans close to optimal solutions and affordable in terms of time and computational burden are highly desirable. The present paper introduces a Logit-based stochastic heuristic approach for finding semi-optimal online contraflow results from the pre-built offline database. With implementing the Logit model, different properties of an offline scenario solution are used for making a decision in the online scenario. Also, the use of stochasticity carrying out Monte Carlo simulation enables us in capturing the unknown features of the problem to some extent. Based on the evaluation results, this method is capable of producing good throughput values that on average are less than $7 \%$ different from the optimal values obtained in a significantly shorter amount of time. The model was calibrated and tested for double breakdowns in the network while it is easily expandable to different combinations of breakdowns due to the flexibility of Logit models. The authors of the paper consider this work as a starting point in a series of studies on trying to understand the capabilities of choice models for connecting the online control problems to the results of the offline control problems. Assuming other choice models, different control strategies will be sought in the future.

\section{Acknowledgements}

The authors remain responsible for the content of this paper. Assistance and support of Professor H. S. Mahmassani for providing us with DYNASMART software are greatly appreciated. Besides, we are deeply grateful to Professor R. Benekohal for reviewing the article.

\section{References}

Afshar, A. M.; Haghani, A. 2008. Heuristic framework for optimizing hurricane evacuation operations, Transportation Research Record 2089: 9-17. doi: 10.3141/2089-02

Birnbaum, Z. W. 1968. On the Importance of Different Components in A Multicomponent System. Technical Report No 54. 20 May 1968. University of Washington. 24 p.

Table 7. Percentage of correct predictions for the final model for calibration and test datasets

\begin{tabular}{|c|c|c|c|c|}
\hline \multirow{2}{*}{ Observation } & \multirow{2}{*}{ Dataset } & \multicolumn{2}{|c|}{ Prediction } & \multirow{2}{*}{ Percentage of correct predictions } \\
\hline & & 0 & 1 & \\
\hline \multirow{2}{*}{0} & Calibration & 2159 & 863 & 71.4 \\
\hline & Test & 2302 & 1800 & 56.1 \\
\hline \multirow{2}{*}{1} & Calibration & 1274 & 3764 & 74.7 \\
\hline & Test & 1554 & 2404 & 60.7 \\
\hline \multicolumn{4}{|c|}{ Total percentage of correct predictions for the Calibration dataset } & 73.5 \\
\hline \multicolumn{4}{|c|}{ Total percentage of correct predictions for the Test dataset } & 58.4 \\
\hline
\end{tabular}


Brown, C.; White, W.; Van Slyke, C.; Benson, J. D. 2009. Development of a strategic hurricane evacuation-dynamic traffic assignment model for the Houston, Texas, region, Transportation Research Record 2137: 46-53. doi:10.3141/2137-06

Campos, V. B. G.; Da Silva, P. A. L.; Netto, P. O. B. 1999. Evacuation transportation planning: A method of identify optimal independent routes, WIT Transactions on the Built Environment (Urban Transport V) 44: 555-564.

Chen, X.; Zhan, F. B. 2008. Agent-based modelling and simulation of urban evacuation: relative effectiveness of simultaneous and staged evacuation strategies, Journal of the Operational Research Society 59: 25-33. doi:10.1057/palgrave.jors.2602321

Chiu, Y.-C. 2004. Traffic scheduling simulation and assignment for area-wide evacuation, in Proceedings of the 7 th International IEEE Conference on Intelligent Transportation Systems, 2004, 537-542. doi:10.1109/ITSC.2004.1398957

Cova, T. J.; Johnson, J. P. 2003. A network flow model for lanebased evacuation routing, Transportation Research Part A: Policy and Practice 37(7): 579-604. doi:10.1016/S0965-8564(03)00007-7

Davis, L. D. 1991. Handbook of Genetic Algorithm. 1 edition. Van Nostrand Reinhold Company. 385 p.

Dunn, C. E.; Newton, D. 1992. Optimal routes in GIS and emergency planning applications, Area 24(3): 259-267.

Kwon, E.; Pitt, S. 2005. Evaluation of emergency evacuation strategies for downtown event traffic using a dynamic network model, Transportation Research Record 1922: 149155. doi:10.3141/1922-19

Lim, E.; Wolshon, B. 2005. Modeling and performance assessment of contraflow evacuation termination points, Transportation Research Record 1922: 118-128. doi: 10.3141/1922-16

Liu, H. X.; Ban, J. X.; Ma, W.; Mirchandani, P. B. 2007. Model reference adaptive control framework for real-time traffic management under emergency evacuation, Journal of Urban Planning and Development 133(1): 43-50. doi: 10.1061/(ASCE)0733-9488(2007)133:1(43)

Mahmassani, H. S. 2000. DYNASMART-P. Volume II. User Guide. Technical Report STO67-85-PII, Center for Transportation Research, The University of Texas at Austin.

Mahmassani, H. S.; Fei, X.; Eisenman, S.; Zhou, X.; Qin, X. 2005. DYNASMART-X Evaluation for Real-Time TMC Application: CHART Test Bed. Final Research Report. Maryland Transportation Initiative, University of Maryland.

Miller-Hooks, E.; Patterson, S. S. 2004. On solving quickest time problems in time-dependent, dynamic networks, Journal of Mathematical Modelling and Algorithms 3(1): 39-71. doi:10.1023/B:JMMA.0000026708.57419.6d

Miller-Hooks, E.; Sorrel, G. 2008. Maximal dynamic expected flows problem for emergency evacuation planning, Transportation Research Record 2089: 26-34. doi:10.3141/2089-04

Noh, H; Chiu, Y.-C.; Zheng, H.; Hickman, M.; Mirchandani, P. 2009. Approach to modeling demand and supply for a short-notice evacuation, Transportation Research Record 2091: 91-99. doi:10.3141/2091-10

Sbayti, H. 2008. Optimal Scheduling of Evacuation Operations with Contraflow: Ph.D. Thesis, University of Maryland, College Park, Maryland, United States. 234 p.

Sbayti, H.; Mahmassani, H. S. 2006. Optimal scheduling of evacuation operations, Transportation Research Record 1964: 238-246. doi:10.3141/1964-26
Smith, J. M. 1991. State-dependent queueing models in emergency evacuation networks, Transportation Research Part B: Methodological 25(6): 373-389.

doi:10.1016/0191-2615(91)90031-D

Szeto, W. Y.; Hong, K. L. 2006. Dynamic traffic assignment: properties and extensions, Transportmetrica 2(1): 31-52. doi:10.1080/18128600608685654

Talebi, K.; Smith, J. M. 1985. Stochastic network evacuation models, Computers \& Operations Research 12(6): 559-577. doi:10.1016/0305-0548(85)90054-1

Tuydes, H. 2005. Network Traffic Management under Disaster Conditions: Ph.D. Thesis, Northwestern University. Illinois, United States. 413 p.

Tuydes, H.; Ziliaskopoulos, A. 2006. Tabu-based heuristic approach for optimization of network evacuation contraflow, Transportation Research Record 1964: 157-168. doi:10.3141/1964-17

Wolshon, B. 2001. 'One-Way-Out': Contraflow Freeway Operation for Hurricane Evacuation, Natural Hazards Review 2(3): 105-112. doi:10.1061/(ASCE)1527-6988(2001)2:3(105)

Wolshon, B. 2002. Planning for the Evacuation of New Orleans, ITE Journal - Institute of Transportation Engineers 72(2): 44-49.

Yuan, F.; Han, L. D.; Chin, S.-M.; Hwang, H. 2006. Proposed framework for simultaneous optimization of evacuation traffic destination and route assignment, Transportation Research Record 1964: 50-58. doi:10.3141/1964-07 